\title{
Simulation Studies of a Trench MOS Device Structure with Small Figures of Merit
}

\author{
Junhong $\mathrm{Li}$ \\ State Key Laboratory of Electronic Thin Films and Integrated Devices, University of Electronic Science and Technology of China
}

\begin{abstract}
We proposed a vertical high permittivity trench power MOS (HKTMOS) device with alternating $\mathrm{N} \& \mathrm{P}$ drift region and high permittivity (HK) trench sandwiched in between. The unique structure guarantees uniform potential distribution for wide voltage range at block state owing to both HK potential modulation effect and superjunction (SJ) charge balance. The specific on-resistance (Rons) of HKTMOS is in orders of magnitude lower than the SJ counterparts at the on state because of the strong accumulation effect brought by HK trench. Although the gate charge also significantly rises because of the accumulation, the figures of merit (FOM) of HKTMOS still reduces considerably than the SJ under same BV. An expression for FOM is derived demonstrating that the FOM of HKTMOS is proportional to the square of HK trench depth, which agrees on with simulation results well. The simulation results indicate that within the BV range of 500 2000V, the Rons and FOM of HKTMOS are in 1 2 orders of magnitude lower and $17.4 \% \sim 44.1 \%$ of SJ, respectively under the same BV condition. Furthermore, HKTMOS also demonstrates better charge imbalance tolerance than SJ.
\end{abstract}

\section{Introduction}

The performance of silicon power devices are essential for the energy conversion system. The introduction of high permittivity (HK) material into power MOS device allows better potential distribution in silicon and therefore improves device breakdown voltage (BV) ${ }^{[1]}$. The effect is called the HK potential modulation (PM) effect $^{[1]}$, which had been demonstrated both theoretically ${ }^{[2]}$ and experimentally ${ }^{[3]}$. On other hand, because HK material exhibits large permittivity, it is potential to be used to activate the carrier accumulation (CA) effect in silicon to significantly reduce the device on-resistance (Ron). In this letter, we propose a novel HK trench MOS (HKTMOS), which utilizes both PM and CA effects from $\mathrm{HK}$, resulting in specific-Ron $\left(\mathrm{R}_{\mathrm{ons}}\right)$ decrease in orders of magnitude compared with conventional superjunction device ( $\mathrm{SJ})$. Although such $\mathrm{R}_{\text {ons }}$ decrease is at the cost of larger switch loss due to the extra accumulation charge on the gate, the relationship between $R_{\text {ons }}$ and gate charge is linear, and the total figures of merit (FOM) of HKTMOS still experiences a significant improvement than the SJ. Furthermore, HKTMOS also indicated strong doping-imbalance tolerance in $\mathrm{N}$ and $\mathrm{P}$ drift region.

\section{Device structure and mechanism}

The cross section view of HKTMOS is showed in Figure 1a. Similar with SJ, HKTMOS also features alternating $\mathrm{N}$ and $\mathrm{P}$ drift region pillar, whereas a layer of $\mathrm{HK}$ material is sandwiched in between. Together with the buried oxide in the $\mathrm{N}+$ drain region, the $\mathrm{P}$ drift region is completely isolated from the $\mathrm{N}$ drift region and the drain, but directly contacts with the gate.

At the device blocking state, with the same mechanism as [4], although there is HK isolation between $\mathrm{N}$ and $\mathrm{P}$ drift region, their electric field still depleted with each other because of the large permittivity of HK, so that the charge balance effect still exists. Moreover, the total permittivity of drift region is also boosted with the introduction of $\mathrm{HK}$ trench, according to [1], the slope of electrical field in drift region is given by (1),

$$
\frac{\partial^{2} \varphi}{\partial^{2} x}=-\frac{\partial E}{\partial x}=-\frac{q N_{d}}{\varepsilon_{\text {total }}},
$$

Where $N_{d}$ and $\varepsilon_{\text {total }}$ are the doping concentration and total permittivity of the drift region, respectively. With large $\varepsilon_{\text {total }}$, the slope of the electric field is small, giving better potential distribution and higher $\mathrm{BV}$ on the basis on charge balance. The effect is attributing to the HK PM effect. As showed in Figure 1b, the potential distribution of HKTMOS is almost ideal at the blocking state. Besides, better doping imbalance tolerance between $\mathrm{N}$ and $\mathrm{P}$ pillar can be also realized with effect of $\mathrm{HK}$, which will be verified later. Above all, at the device blocking state, both effect of HK PM and charge balance co-exists to guarantee the high BV not only for the short drift region, but also for long drift region devices. 


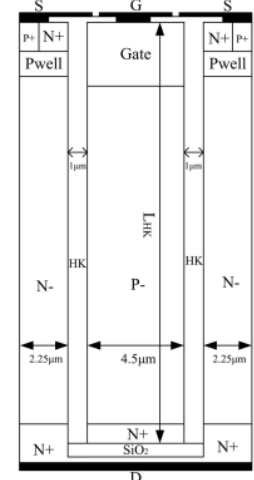

(a)

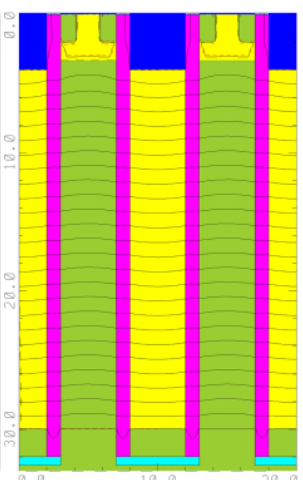

(b)

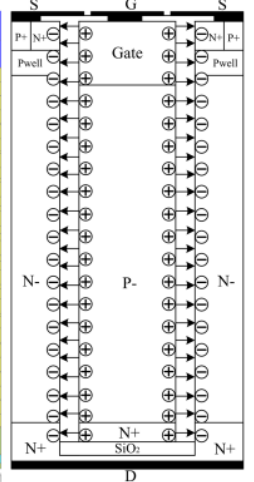

(c)
Fig 1. (a) HKTMOS cross section view with $\mathrm{N}$ - and $\mathrm{P}$ - doping of $10^{-15} \mathrm{~cm}^{-3}$. (b) Potential distribution of HKTMOS at block state. (c) Illustration of the accumulation effect.

Although the reduction of device $\mathrm{R}_{\text {ons }}$ by mean of CA effect has been reported in [5], the CA is generated by $\mathrm{SiO}_{2}$ layer, with the low permittivity of $\mathrm{SiO}_{2}$, its effect is limited. Moreover, small $\varepsilon$ of the $\mathrm{SiO}_{2}$ also brings negative effect for BV according to (1). On contrast, HK material is capable of overcoming the above problems. When the device in Figure 1a is in on state, the gate voltage is high, the $\mathrm{P}$ pillar share the same high potential with gate as their directly contact. Whereas both drain and source voltage is much lower than the gate at the on state, consequently, CA effect happens at the interface of silicon and HK trench as showed in Figure 1c. The large permittivity of $\mathrm{HK}$ amplified the CA effect and forms a high carrier density path between drain and source, the device $\mathrm{R}_{\text {ons }}$ is thereby significantly reduced.

CA effect allows significant $R_{\text {ons }}$ reduction with no additional drift region doping. However, large trench capacitance is necessary to accumulate enough charge at the device on-state to reduce the $\mathrm{R}_{\text {ons. And the trench }}$ capacitor will be discharged at off-state to guarantee high BV, such charge-discharge cycle with large capacitance results in high switch loss. Rarely had any literatures qualitatively investigated such effect and its impact to FOM. For the HKTMOS device, as a low resistance path exists between drain and source generated by $\mathrm{HK} C A$ effect, the device $R_{\text {ons }}$ is major determined by the accumulation resistance $\left(\mathrm{R}_{\mathrm{A}}\right)$. According to [6], $R_{A} \propto 1 / C_{g}$, where $C_{g}$ is the unit area

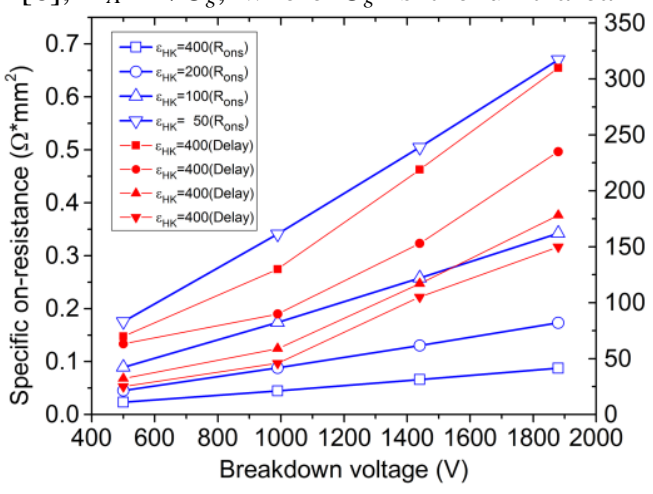

(a)

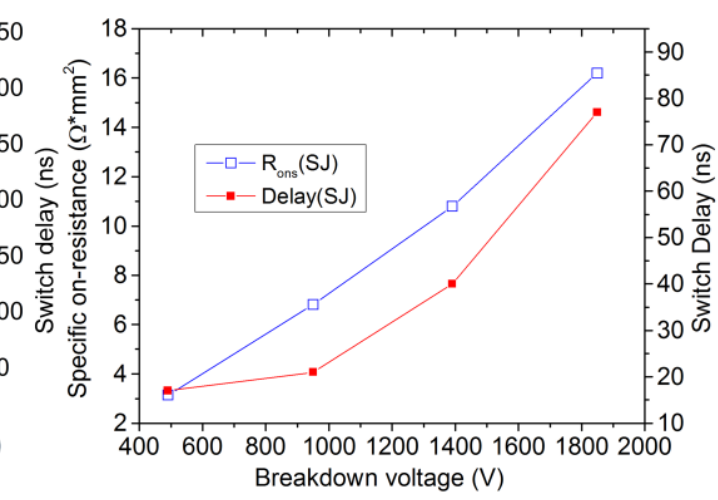

(b) trench capacitance, we can derive that $\mathrm{R}_{\text {ons }}$ of HKTMOS is given by

$$
R_{\text {ons }}=\frac{K_{H K T} L_{H K}}{V_{g} C_{g} W}
$$

Where the $K_{H K T}, L_{H K}, V_{g}$, and $W$ are the HKTMOS constant, depth of HK trench, gate voltage and width of the device, respectively. Although the $\mathrm{N}+$ source junction and the drain not contributing to the total resistance are covered in the $L_{H K}$ in (2), both of their depth are overwhelming smaller than the drift region length and thereby negligible. We see that the $R_{\text {ons }}$ is proportional to $L_{H K}$ for HKTMOS from (2).

On the other hand, the gate charges are contributed by both $\mathrm{HK}$ trenches and $\mathrm{SiO}_{2}$ buries. The contribution from $\mathrm{SiO}_{2}$ buries is also overwhelming smaller than the HK trenches, for both permittivity and area. Therefore, it is neglected and gate charge is given by

$$
Q_{g}=W L_{H K} V_{g} C_{g}
$$

According to (2) and (3), we see that the $R_{\text {ons }}$ is inverse proportional to the gate charge for a given device dimension. The definition ${ }^{[6]}$ for FOM is $F O M=R_{\text {ons }} * Q_{g}$, which gives

$$
F O M_{H K T}=K_{H K T} L_{H K}^{2}
$$

An interesting result is observable from (4) that FOM of the HKTMOS is irrespective of the unit area trench capacitance and device width. It is only proportional to the square of the $\mathrm{HK}$ trench depth with ratio of HKTMOS constant $K_{H K T}$. According to analysis and simulation results, the analytical value of $\mathrm{K}_{\mathrm{HKT}}$ is $7.812 \times 10^{-4} \Omega * \mathrm{nC} / \mu \mathrm{m}^{2}$.

\section{Simulation verification and discussion}

We use TMA-MEDICI to make 2D simulation and verify above analysis. Figure 2 shows the BV dependence for $\mathrm{R}_{\mathrm{ons}}$ and switch delay for both HKTMOS with different HK permittivity $\varepsilon_{H K}$ (a) and SJ device (b). Fig 2. Under the $15 \mathrm{~V}$ gate voltage, the $\mathrm{R}_{\mathrm{ons}} \mathrm{vs.} \mathrm{BV}$ and switch delay (sum of the transient rise and fall time for the drain voltage) vs
$\mathrm{BV}$ with a drain resistance of $4 \times 10^{6} \Omega$ for HKTMOS (a) and $4 \times 10^{7} \Omega$ for SJ (b) under the dimension of $20 \mu \mathrm{m} \times 1 \mu \mathrm{m}$ for both devices. The Rons and the switching delay are simulated under a drain voltage of $0.1 \mathrm{~V}$ and $100 \mathrm{~V}$, respectively. 
As revealed in Figure $2 b$, the $R_{\text {ons }}$ of the $S J$ rises rapidly with increase of $\mathrm{BV}$ at the exponential of 1.33[4]. On contrast, under the effect of strong CA effect brought by $\mathrm{HK}$, the $\mathrm{R}_{\text {ons }}$ of HKTMOS is in the orders of magnitude lower than the SJ as showed in Figure 2a. Higher $\varepsilon_{H K}$ always brings smaller $R_{\text {ons }}$, owing to the reason that higher permittivity always provides larger trench capacitance, thereby stronger CA effect and smaller $R_{\text {ons. }}$. Moreover, expression (2) suggests that the $R_{\text {ons }}$ of HKTMOS is proportional of the drift region length. Because the BV is also proportional to the drift region length, the $R_{\text {ons }}$ of HKTMOS rises linearly with the increase of $\mathrm{BV}$ as Figure $2 \mathrm{a}$ reveals, which agrees on with (2).

Although the CA effect of HK reduces $\mathrm{R}_{\text {ons }}$ in orders of magnitude, the large trench capacitance also slow down the switching speed. As showed in Figure 2, the switch delay of the HKTMOS is much larger than the SJ device, in the orders of magnitude as well. Higher $\varepsilon_{H K}$ value always causes worse switch delay. To investigate the comprehensive performance of the HKTMOS, the device FOMs are simulated and calculated as shown in Figure $3 \mathrm{a} . \mathrm{R}_{\mathrm{ons}}$ is simulated under the condition of a $15 \mathrm{~V}$ gate voltage and $0.1 \mathrm{~V}$ drain voltage. $\mathrm{Q}_{\mathrm{g}}$ is simulated schematically as shown in Figure $3 \mathrm{a}$, where a $15 \mathrm{~V}$ square wave voltage is applied on the gate. $\mathrm{Q}_{\mathrm{g}}$ is

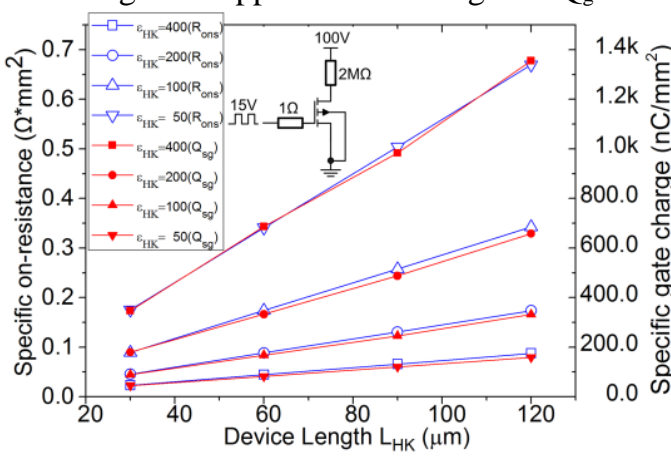

(a) determined by the current integral at the time interval when the drain drops from $100 \mathrm{~V}$ to the voltage of the fully on-state. As Figure $3 \mathrm{a}$ shows, both $\mathrm{R}_{\mathrm{ons}}$ and $\mathrm{Q}_{\mathrm{g}}$ increase linearly with the rise of $\mathrm{L}_{\mathrm{HK}}$, which is consistent with (3) and (5). (3) and (5) also indicated that $R_{\text {ons }}$ and $\mathrm{Q}_{\mathrm{g}}$ are proportional and inversely proportional to $\varepsilon_{H K}$, respectively. The relation is also verified by simulation, as Figure 3a shows; a larger $\varepsilon_{H K}$ always provides a smaller $R_{\text {ons }}$ but a larger $Q_{g}$ and vice versa.

Figure $3 b$ shows the FOM and BV vs. $\mathrm{L}_{\mathrm{HK}}$ for both HKTMOS and SJ, where the FOM of HKTMOS with changing $\varepsilon_{\mathrm{HK}}$ are plotted in different line styles. As mentioned above, $\mathrm{R}_{\mathrm{ons}}$ and $\mathrm{Q}_{\mathrm{g}}$ are inversely proportional and proportional to $\mathrm{L}_{\mathrm{HK}}$, respectively, which cancel each other out when the multiplication for FOM occurs. It can be observed that all of the lines almost overlap with each other for different $\varepsilon_{H K}$ when $\mathrm{L}_{\mathrm{HK}}$ ranges from $30 \mu \mathrm{m}$ to $120 \mu \mathrm{m}$ (Figure 3b) according to simulation, which agrees with (6) very well. The proportional coefficient between the FOM and $\mathrm{L}_{\mathrm{HK}}$ square may be different depending on the cell geometry; however, for a device with a given cell geometry, the FOM is only determined by $\mathrm{L}_{\mathrm{HK}}$ regardless of the $\mathrm{HK}$ permittivity and trench thickness.

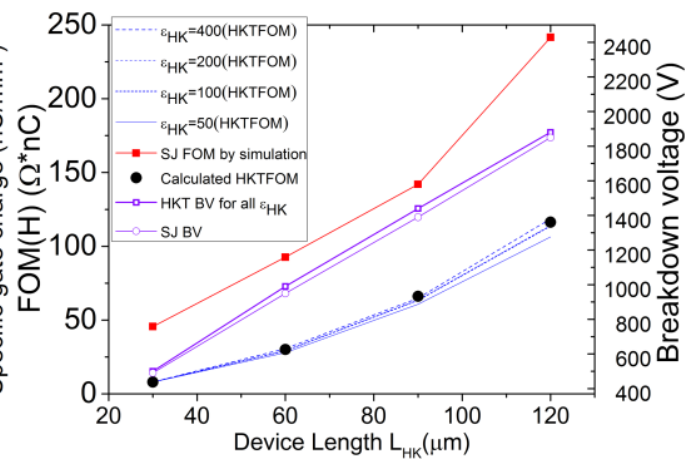

(b)

Fig 3. The $R_{\text {ons }}$ (simulated under $15 \mathrm{~V}$ gate voltage and $0.1 \mathrm{~V}$ drain voltage) and $\mathrm{Q}_{\mathrm{g}}$ (simulated by the integral of the gate current as the schematics show in the figure) vs. LHK for HKTMOS (a) The FOM(H) vs. LHK for HKTMOS by both simulation and calculation, together with the $\mathrm{FOM}(\mathrm{H})$ of SJ and BV by simulation (b).

For HKTMOS with the size displayed in Figure 2a, the analytical value of the proportional coefficient is derived as $7.812 \times 10^{-3} \Omega * n C / \mu m^{2}$. Utilizing such a coefficient, we are able to calculate the FOM using (6), which is shown by the round dots in Figure $3 \mathrm{~b}$. It is clear that the calculation results agree well with the simulation results. Figure $3 \mathrm{~b}$ also reveals that for the HKTMOS with smaller $\varepsilon_{\mathrm{HK}}\left(\varepsilon_{H K}=50\right)$, the FOM by simulation is slightly smaller than the calculated value, especially at larger $\mathrm{L}_{\mathrm{HK}}$. This is because $\mathrm{Q}_{\mathrm{g}}$ is determined by the sum of $\mathrm{Q}_{\mathrm{HK}}$ and $\mathrm{Q}_{\mathrm{DC}}$, then, $\mathrm{Q}_{\mathrm{g}}$ will drop linearly with the linear decrease of $\varepsilon_{H K}$ as long as $\mathrm{Q}_{\mathrm{HK}}$ is still larger than QDC according to (4). However, as $R_{A}$ parallels with $R_{\text {drift }}$, with smaller $\varepsilon_{H K}, R_{A}$ in (2) is less dominant, and the contribution to $R_{\text {ons }}$ from the drift region $R_{\text {drift }}$ becomes more significant although $R_{A}$ is still much lower than $R_{\text {drift }}$. Consequently, the total $R_{\text {ons }}$ will rise sub-linearly with the decrease of the small $\varepsilon_{H K}$ due to the contribution from the drift region bypass, which has been neglected in previous analysis. Moreover, according to (6), the FOM of HKTMOS is proportional to the square of $L_{H K}$; the square amplifies the model inaccuracy if $L_{H K}$ is large, which causes a larger FOM difference between the calculated and simulated values under the condition of a large $\mathrm{L}_{\mathrm{HK}}$ and a small $\varepsilon_{H K}$ as shown in Figure $3 \mathrm{~b}$.

Figure $3 \mathrm{~b}$ also shows the relationship between BV and $\mathrm{L}_{\mathrm{HK}}$ for both HKTMOS and SJ. The BVs of HKTMOS with different $\varepsilon_{H K}$ completely overlapped with each other so that only one curve is shown for HKTMOS. As the BV of SJ relies on $\mathrm{CB}$ only, while HKTMOS takes both effects of PM and $\mathrm{CB}$ to achieve high $\mathrm{BV}$, the $\mathrm{BV}$ of HKTMOS will be slightly better than that of SJ, as shown in Figure 3b. Last but not least, HKTMOS always demonstrates significant FOM improvement over that of SJ under all $\mathrm{L}_{\mathrm{HK}}$ in the figure; the FOM of HKTMOS is $48 \%$ of that of SJ at the $\mathrm{L}_{\mathrm{HK}}$ of $120 \mu \mathrm{m}$, and only $17 \%$ at the $\mathrm{L}_{\mathrm{HK}}$ of $30 \mu \mathrm{m}$. Although the FOM takes the total gate charges into consideration instead of the gate-to-drain charges only, HKTMOS still exhibits a significant FOM improvement over that of SJ. 
Charge imbalance between $\mathrm{N} \& \mathrm{P}$ drift region in actual fabrication process is another issue of the SJ, even slight charge imbalance caused by doping difference can severely impact the BV of SJ device. Adversely, HKTMOS exhibit excellent doping difference tolerance because their BVs not only depend on charge balance but also PM effect from HK trench. As Figure 4 shows, with the rise of the N\&P drift region doping difference, the BV of HKTMOS with $\varepsilon_{H K}=400$ almost remains unchanged. However, smaller $\varepsilon_{H K}$ value always causes higher BV sensitivity to doping difference, this is because with smaller $\varepsilon_{H K}$, the PM capability of $\mathrm{HK}$ trench is impaired, consequently, the BV drops more rapidly with the rise of doping difference. As the BV of SJ solo relies on the charge balance and free of PM effect, SJ gives the worst performance. The BV of SJ at the doping difference of $4 \pm 10^{-16} \mathrm{~cm}^{-3}$ drops to $30 \%$ of the $\mathrm{BV}$ under the charge-balance condition.

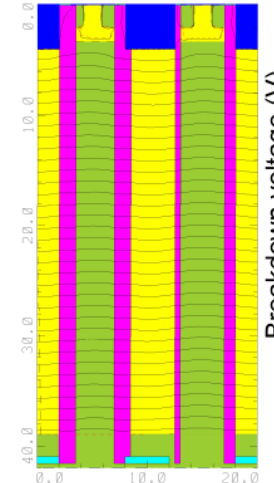

(a)

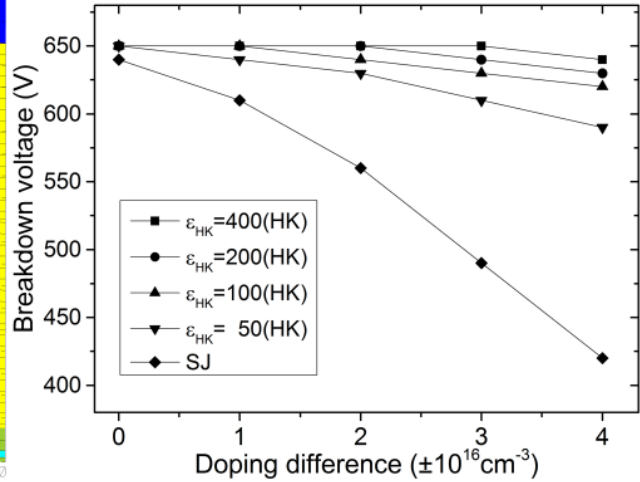

(b)
Fig 4. (a) The potential distribution for a HKTMOS with $\mathrm{L}_{\mathrm{HK}}$ of $39 \mu \mathrm{m}$ and HK trench randomly shifted in both width and position. (b) The impact of charge-imbalance to BV for SJ and HKTMOS with different $\varepsilon H K$ for the device length of $39 \mu \mathrm{m}$ under the baseline doping of $10^{15} \mathrm{~cm}^{-3}$ for both $\mathrm{N}$ and $\mathrm{P}$ pillar.

\section{Conclusion}

The HKTMOS we proposed in this letter indicates excellent performance for wide $\mathrm{BV}$ range according to investigation. Its FOM is predictable using the HKTMOS constant and shows significant improvement than that of the SJ. Besides, HKTMOS also demonstrates its good charge-imbalance tolerance than its SJ counterparts. Above all, the HKTMOS is potential for the application on the energy conversion system for better efficiency and further promotion of the silicon limit.

\section{References}

1. X. Chen, "Super-junction voltage sustaining layers with alternating semiconductor and high-K dielectric regions," U.S. Patent, 7230310B2, Jun. 12, 2007

2. X. Luo, Y. Jiang, K. Zhou, P. Wang, X. Wang, Q. Wang, G. Yao, B. Zhang, and Z. Li, "Ultralow specific on-resistance superjunction vertical DMOS with high-K dielectric pillar," IEEE Electron Device Lett., vol. 33, no. 7, pp. 1042-1044, Jul. 2012.

3. J. Li P. Li, W. Huo, G. Zhang, Y. Zhai, and X. Chen, "Analysis and fabrication of an LDMOS with highpermittivity dielectric," IEEE Electron Device Lett., vol. 32, no. 9, pp. 1266-1268, Sep. 2011.

4. X. Chen and J. Sin. "Optimization of the specific on-resistance of the COOLMOS (TM)," IEEE Trans. Electron Devices, vol. 48, no. 2, pp. 344-348, Feb. 2001.

5. B. J. Baliga, T. Syau, and P. Venkatraman, "The accumulation-mode field effect transistor: a new ultralow on-resistance MOSFET" IEEE Electron Device Lett., vol. 13, no. 8, pp. 427-429, Aug. 1992.

6. B. J. Baliga, "Fundamentals of power semiconductor devices," Springer-Verlag, 2008, pp. 362.

7. B. J. Baliga, "Advanced power MOSFET concepts," Springer-Verlag, 2010, pp. 55. 